\title{
Development and Deployment of an Intraoperative Clinical Summary Document: A Veterans Health Care Affairs Perspective
}

\author{
Seshadri C. Mudumbai ${ }^{1,2,3}$ Troy Tanaka ${ }^{4}$ Edward R. Mariano ${ }^{1,2}$ \\ ${ }^{1}$ Anesthesiology and Perioperative Care Service, Veterans Affairs Palo \\ Alto Health Care System, Palo Alto, California, United States \\ 2 Department of Anesthesiology, Perioperative and Pain Medicine, Stanford \\ University School of Medicine, Stanford, California, United States \\ ${ }^{3}$ Center for Innovation to Implementation, Veterans Affairs Palo Alto \\ Health Care System, Menlo Park, California, United States \\ ${ }^{4}$ VISN 21 CIS/ARK Service, Veterans Affairs Palo Alto Health Care \\ System, Palo Alto, California, United States \\ ACI Open 2020;4:e30-e34.
}

Address for correspondence Seshadri C. Mudumbai, MD, MS, Anesthesiology and Perioperative Care Service, 112A, VA Palo Alto HCS, 3801 Miranda Avenue, Palo Alto, CA 94304, United States (e-mail: mudumbai@stanford.edu).

\section{Abstract}

Keywords

- intraoperative clinical summary documents

- clinical summary

- provider

- health records

- anesthesiology

- quality improvement

- anesthesia information management system
Background Clinical summary documents that abstract details of episodes of care are essential to Advancing Care Information requirements for electronic health records. Few examples exist for intraoperative clinical summary documents (ICSD).

Methods We describe the development and deployment of an ICSD at a tertiary hospital within the United States. Development included identification of needs of key stakeholders, evaluation of current clinical and data workflows, iterative development of prototypes with primary stakeholders, i.e., anesthesiologists and evaluation of prototypes with test patients. We deployed the ICSD over 6-months with tracking of (1) usage (number and types of end users and surgery types) and (2) written and oral feedback.

Results Current workflows involved accessing a 10 to 40 page document presenting all surgery details with review described as burdensome. The ICSD prototype was a separate one-page summary optimized for viewing on a monitor or paper. The document had four sections: (1) administrative data, allergies, and precautions; (2) medications, infusions, and fluid intake and output; (3) airway assessments and a graphical presentation of hemodynamic trends (blood pressure), and (4) standardized text for events (hypotension) occurring intraoperatively. During the 6-month deployment, postanesthesia care unit and intensive care unit nurses were most prominent users followed by anesthesiologists. While overall well received, our pilot users reported challenges for readability, font size, and the lack of customization.

Conclusion While the ICSD was designed for anesthesiologists, postanesthesia care unit and intensive care unit nurses were the most frequent users. Future development will involve customization for different stakeholders. received

March 20, 2019

accepted after revision

December 16, 2019
DOI https://doi.org/

10.1055/s-0040-1701192.

ISSN 2566-9346. (c) 2020 Georg Thieme Verlag KG

Stuttgart · New York
License terms

()(1) 


\section{Background and Significance}

With the proliferation of electronic health records (EHR), large volumes of data are being archived. ${ }^{1}$ Within the United States, generating clinical summary documents that abstract details of an episode of care is essential to Advanced Care Information (ACI) requirements as part of the Merit-Based Incentive Programs (MIPS). ${ }^{2,3}$ While automated summarization approaches have been studied, their effectiveness is variable, and the need for customized summaries that directly support clinical workflows persists. ${ }^{4,5} \mathrm{~A}$ particular example where the need for providing summaries is crucial is the perioperative environment. The operating room is characterized by complex care patterns, a need for extensive planning both before and after surgery, and generation of large volumes of physiologic data (e.g., heart rate). ${ }^{6}$ However, few examples exist within the published literature of intraoperative clinical summary documents (ICSD).

The Veterans Affairs (VA) health care system is the largest networked health care system in the United States. Anesthesiologists at a tertiary care VA hospital reported challenges accessing and evaluating intraoperative records due to the lack of an ICSD. We therefore sought to design, implement, and evaluate a Windows 7-compliant ICSD that helps providers understand key events, medications, and patient characteristics from a surgical episode of care. The aim of this quality improvement report is to present our experience in the development and deployment of the ICSD relative to prior records.

\section{Methods}

\section{Organizational and Technical Setting}

This quality improvement project took place at a universityaffiliated tertiary care VA hospital that conducts both inpatient and outpatient surgical procedures (i.e., general surgery, neurosurgery) with 2,000 procedures annually. The facility has hosted an integrated operating room, postanesthesia care unit, and intensive care unit perioperative information management system (PIMS) since 2017: Picis 8.2 (Wakefield MA). The PIMS demonstrates interoperability with the larger VA enterprisewide EHR, VistA (Veterans Health Information Systems and Technologies Architecture). ${ }^{7}$ The PIMS receives demographic and admission-related information (standard HL7 interfaces) with VistA and combines clinical data from monitoring devices (e.g., electrocardiogram).

\section{Development of the ICSD}

The development of the ICSD involved (1) identification and needs of key clinical stakeholders; (2) evaluation of current clinical and data workflows; (3) iterative development of prototypes with primary stakeholders, i.e., anesthesiologists and the PIMS vendor; and (4) evaluation of prototypes with a series of test patients.

First, we identified types and numbers of potential, clinical stakeholders. Based on requests from facility leadership and discussions with anesthesiologists for development of ICSD, primary stakeholders were designated to be anesthesiologists $(n=11)$. To identify other stakeholders, we evaluated staff rosters and usage logs. Annually, approximately 150 staff including nurses, physicians (surgeons/intensivists), and allied personnel used the PIMS: perioperative nurses (postanesthesia care $[n=20]$; intensive care unit $[n=70]$ ); quality improvement personnel $(n=10)$; respiratory therapists $(n=10) ; \quad$ surgeons/intensivists $\quad(n=15)$; pharmacists $(n=10)$; and managers $(n=2)$. It was noted that perioperative nurses would have a vested interest in an ICSD.

Second, we evaluated current clinical and data workflows. The development team (one nursing and one physician informaticist) met regularly over a 1 -year period with anesthesiologists, nursing representatives, and local informatics personnel. Across all users, clinical workflows involved accessing a 10 to 40 page electronic portable document format (PDF) document stored within VistA Imaging (see below). End users routinely described review of this document as burdensome and timeconsuming. The PDF was difficult to review because it presented all physiologic output (in 1 or 5 -minute intervals), a comprehensive listing of medications, and intraoperative events. Rows were often blank (i.e., because no medications were administered) causing difficulties in reading. Primary stakeholders requested a one-page summary document separate and different from the electronic anesthesia record that incorporated key elements such as medications or visual summaries of physiologic data. The goal was not a redesign of the graphical user interface.

Regarding data flows, to generate the current PDF, PIMS data was directly imported into VistA-imaging, an image management system. In VistA-Imaging, application entities use DICOM (Digital Imaging and Communications in Medicine) frameworks to receive, store, and make accessible images through user-driven utilities. ${ }^{8}$

Third, we engaged in iterative development cycles with anesthesiologists and the vendor. This development process involved face-to-face and phone interviews and email discussions with a self-selected group of three anesthesiologists and the anesthesia chief (who provided sustained feedback throughout the development process). Discussions revolved around necessary components for a one page ICSD (e.g., medications, physiologic data). Paper mock-ups involving the components and potential layout were created. Once a satisfactory paper version was created, digital ICSD versions were developed with the vendor's help. Four cycles of development occurred incorporating feedback for potential usability, content, and layout.

Fourth, after a near-final digital prototype ICSD was developed, test patients were developed that had fictional physiologic, medication, and event data. Twenty real patients' historical data were also evaluated for fidelity and accuracy of data abstraction for a wide variety of surgeries (e.g., ophthalmology, general surgery). Site-specific information such as server paths were added prior to batch file updates immediately prior to deployment.

\section{Pilot Deployment with Evaluation}

We deployed the ICSD over a 6-month interval and tracked deployment both by usage metrics as well as feedback. Our usage metrics included total number of times the ICSD was 

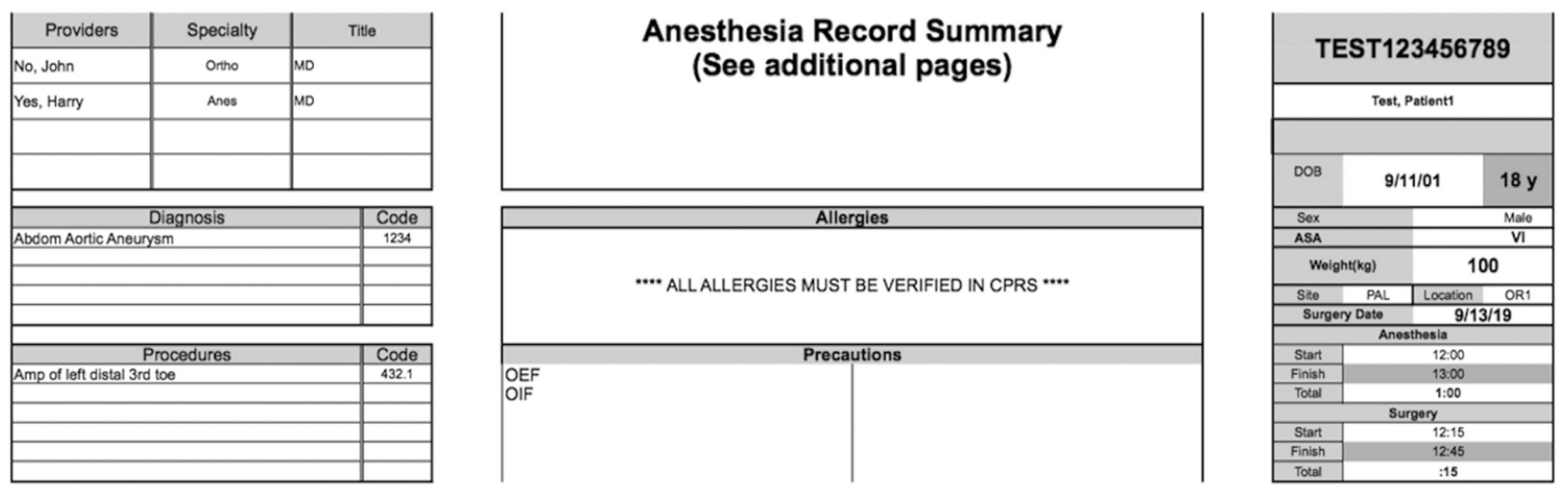

Fig. 1 Sections of the intraoperative clinical summary document (ICSD). Panel series show sections of the ICSD. The ICSD was intended primarily for viewing on a monitor. Section 1-administrative data (providers, diagnosis, procedures) are to the left. Allergies and precautions (e.g., suicide risk) are shown prominently in the middle box. Patient name and key identifiers (social security number), and procedure information (e.g., start time) are to the right.

accessed, usage by type of stakeholder (e.g., anesthesiologists; nursing, informatics personnel; laboratory, pharmacy, biomedical, surgeons, quality assurance personnel, patient safety, other), and by type of surgery. We also noted whether any failures were found in creation of printouts. All other data were qualitative and unstructured. We collected both in-person and e-mailed reports of what worked well and what did not, and summarized feedback into broad themes.

\section{Results}

The prototype was built as a one-page summary document, separate from the electronic anesthesia record, with four sections that could be readily viewed either on a monitor or printed. The sections were as follows
(1) administrative data (e.g., name, procedure, providers), allergies and precautions (e.g., suicide risk); (2) medications (bolus); infusions (e.g., vasopressors), and fluids intake and output (e.g., blood loss); (3) airway assessments; and a graphical presentation of hemodynamic trends (e.g., blood pressure, heart rate); (4) standardized text for events (e.g., cardiopulmonary resuscitation) and assessments (e.g., hypotension) occurring intraoperatively (-Figs. 1-4).

Overall, users accessed the summary report 1,460 times during the 6-month trial. We noted usage across all stakeholders with the most frequent among clinical stakeholders being nursing (29.6\%), nonclinical other (13.9\%), and anesthesiologists (7.5\%). Cases were accessed for all surgery types. Informatics personnel often assisted end users in accessing the ICSD. Feedback themes were noted: anesthesiologists found

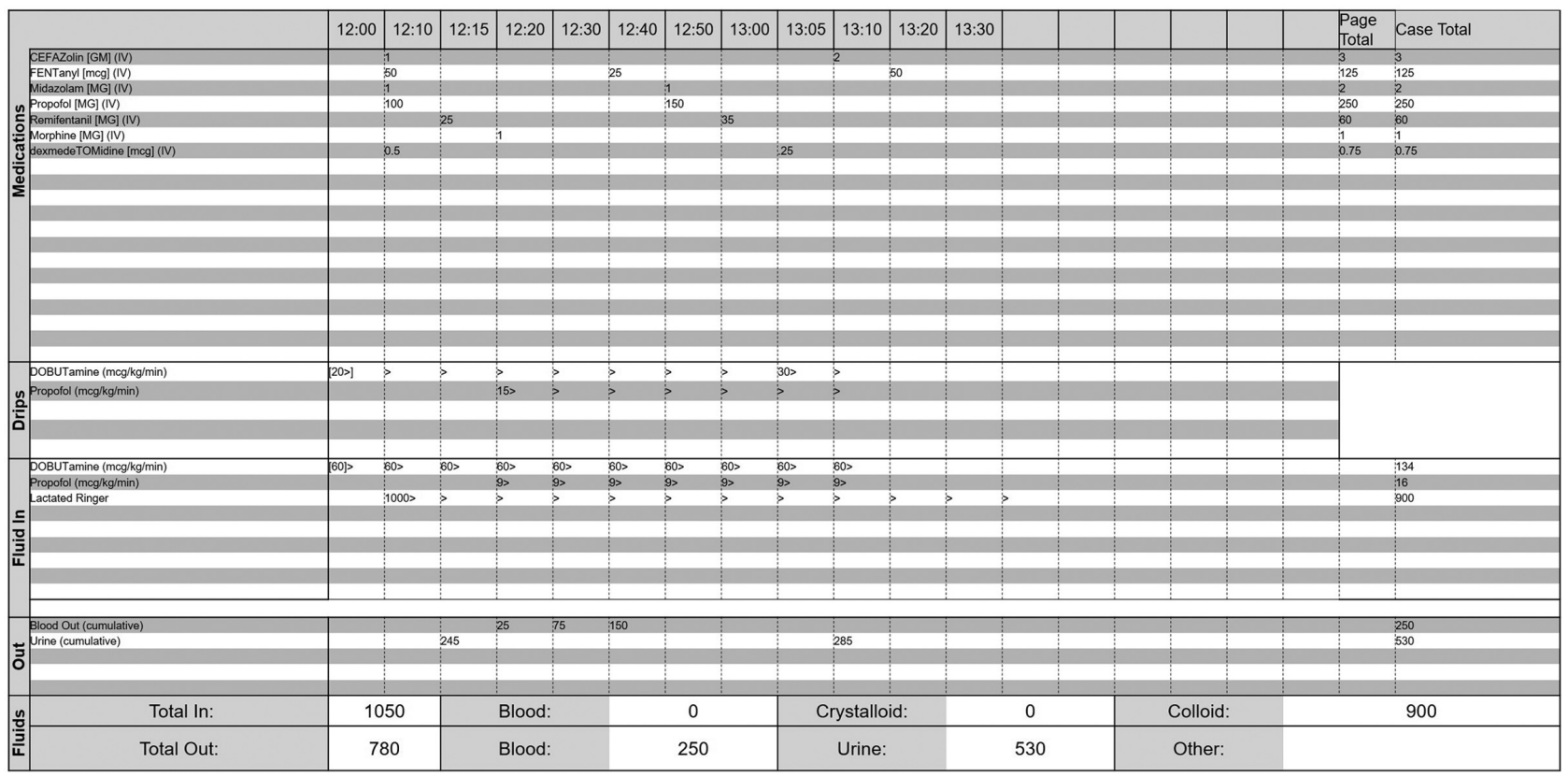

Fig. 2 Sections of the intraoperative clinical summary document (ICSD). Panel series show sections of the ICSD. The ICSD was intended primarily for viewing on a monitor. Section 2-medications (bolus); infusions (e.g., vasopressors), and fluids intake and output (e.g., blood loss) are shown to the left. All data (doses, fluid volumes) were time correlated with totals presented to the right of the panel as appropriate. 


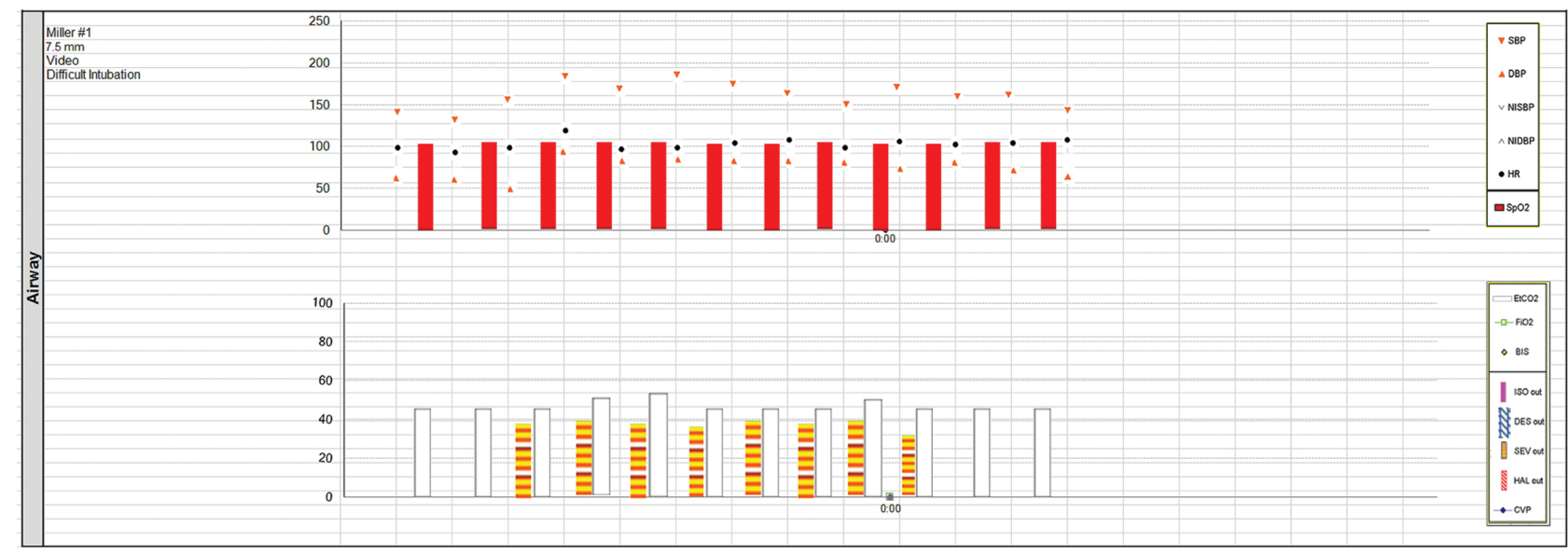

Fig. 3 Sections of the intraoperative clinical summary document (ICSD). Panel series show sections of the ICSD. The ICSD was intended primarily for viewing on a monitor. Section 3-Airway assessments (e.g., type of laryngoscope) are to the left. Then two graphical subsections are displayed. On top are presentations of hemodynamic trends. Elements displayed here include systolic, diastolic, and mean blood pressures; heart rate; and oxygen saturation. On bottom are trends in end tidal carbon dioxide, fraction of inhaled oxygen, bispectral index, and inhalation gases.

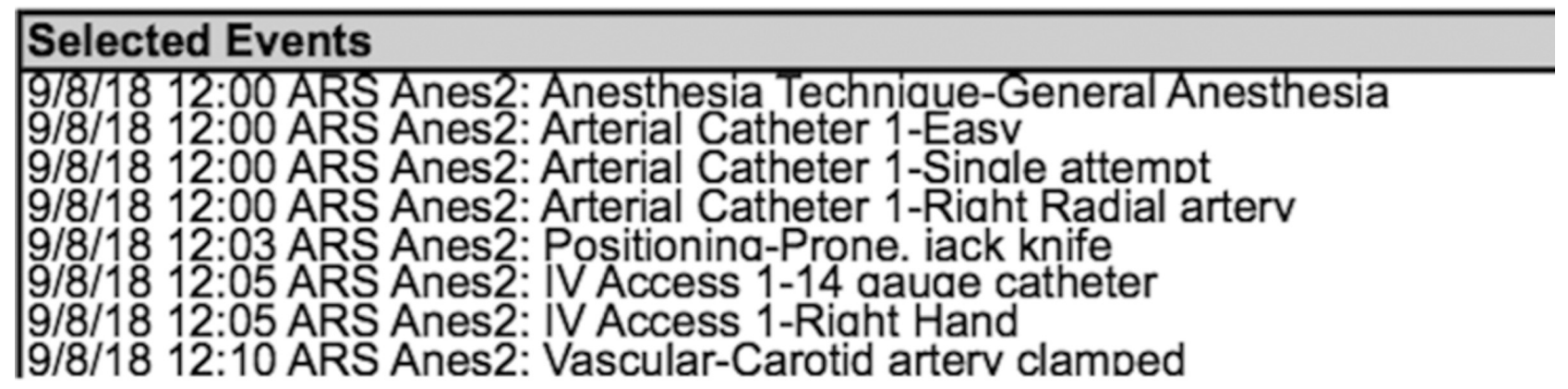

Fig. 4 Sections of the intraoperative clinical summary document (ICSD). Panel series show sections of the ICSD. The ICSD was intended primarily for viewing on a monitor. Section 4-standardized text for events (e.g., cardiopulmonary resuscitation) and assessments (e.g., hypotension) occurring intraoperatively is shown.

the ICSD helpful for anesthetic plan preparation and nurses noted ICSD aided review of postsurgical care. However, concerns were raised about lack of readability upon printing (i.e., need for larger fonts and lack of customizability of different sections for nonanesthesiologist stakeholders).

\section{Discussion}

In this report of a quality improvement effort, we describe the development of an ICSD and pilot deployment in a busy, tertiary referral hospital. The ICSD was separate from the overall electronic anesthesia record. A one-page, printable summary that presented administrative data, overall hemodynamics, trends, key assessments, and administered medications was found essential to summarizing records for anesthesiologists and nurses. Based on usage statistics and the qualitative reports we elicited, postanesthesia care and intensive care unit nurses accessed the ICSD most frequently. Anesthesiologists did note that the ICSD addressed the main use case: preparation of anesthetic plans for surgical patients who received an anesthetic previously. Few published reports of anesthesia summary documents exist and that incorporate meaningful use with usage statistics and user feedback. In addition, our case report promotes generalizability; perioper- ative summary documents can be useful to summarize medications and perioperative events that are typically a "black box" to clinicians outside of perioperative settings.

Our pilot effort is relevant to the need for clinical summaries as required by $\mathrm{ACI}$ components of the MIPS requirements for EHRs. ${ }^{2,3,9}$ Similar to prior meaningful use requirements, clinicians and health care systems are required to provide summaries of care. Our pilot effort helps to address intraoperative episodes of care. No summary documents existed for the intraoperative period prior to this effort. Our ICSD documents are stored in centralized servers for VistA Imaging ${ }^{8}$ and so may be accessible throughout the VA health care system. ${ }^{7}$ The ability to present key features from previous surgical episodes may be helpful in reducing cognitive workloads for anesthesiologists and nurses, and enhance patient safety, particularly during transfers of care from operating rooms to intensive care unit. ${ }^{0,11}$ Our data indicated that summaries were accessed across all surgery types, including those with potentially higher intraoperative workloads such as neurosurgery.

While anesthesiologists were intended to be primary users, among clinical stakeholders, we found instead that nurses from postanesthesia care and intensive care units accessed the ICSD most often. The postanesthesia care unit nurses noted that they found the ICSD useful because it 
helped to present a quick summary of patient surgical care. The information presented in the ICSD allowed them to identify nuances of intraoperative care such as trends of blood pressure changes or blood loss and thereby aid in medication choices for hemodynamic management. Qualitative feedback also indicated that the focus group anesthesiologists $(n=3)$ were willing to incorporate and access the ICSD routinely in their preoperative planning. However, the larger group of anesthesiologists $(n=7)$ felt the use of the ICSD was warranted mainly in patients with a complicated medical history (and a more difficult intraoperative course) or an adverse outcome (e.g., allergic reaction). Since the facility was a tertiary referral center with seriously ill patients presenting for surgery, this finding suggests that we could have improved utilization by anesthesiologists by highlighting the usefulness of the ICSD in planning for patients with high acuity. While practitioners may need summaries and the ability to access key events, departments also need the tools to review compliance with regulatory standards. An example is administration of antibiotics 1 hour prior to surgical incision. ${ }^{12}$ Our pilot study indicated that a variety of end users did access the report including staff from Pharmacy and Quality Improvement.

Based on our observations and informal internal team feedback, we see future development occurring as follows. First, ICSDs could be developed specifically designed for other stakeholders. While the current version is primarily designed for use by anesthesiologists, future versions could create summary documents tailored to the needs of pharmacists and quality improvement staff. This might involve medication summaries of opioid consumption for example. Second, development could address the need for visual, graphical summaries for content like intraoperative medications or assessments. This could involve creation of bar plots (e.g., percent of assessments with a negative outcome like nausea or vomiting) or more complex representations like heat maps. Heat maps have been used to visualize relationships between two domains like medication adherence and vital signs in the setting of acute myocardial infarction. ${ }^{13}$ Similarly, the capacity to visualize relationships between intraoperative medications and changes in blood pressure may be of use in anesthetic planning. Finally, the information gained in creating the ICSD could be helpful to modify the main electronic anesthetic record. For example, we could better highlight key documentation elements using color schemes or fonts to enhance readability. Documentation elements specific and recognized as important to our facility would include medications (bolus), infusions like vasopressors, and standardized text for events (e.g., cardiopulmonary resuscitation) and assessments (e.g., hypotension) that may have occurred intraoperatively.

Limitations to our pilot effort include that we did not measure how clinical management, (i.e., anesthetic plans) was modified as a result of accessing the summary. We also did not measure which individual elements within the ICSD were accessed. However, our goal was to report our experience in the development of this tool and on its initial deployment. This pilot effort also occurred within a VA system and therefore may not be applicable to non-VA settings but has potential generalizability across VA.

In summary, after an iterative development process, we deployed an ICSD as a pilot effort. Initially well received by nurses and anesthesiologists, enhancements could focus on addressing individual use cases and visual analytics to further enhance incorporation into clinical workflows.

\section{Clinical Relevance Statement}

This work is relevant to the clinician as an ICSD has the ability to enhance the workflow of anesthesiologists, nurses, pharmacists, and quality improvement staff. The document was deployed as a pilot effort at a tertiary referral VA hospital, underwent wide usage, and has potential relevance to a diverse set of clinical users.

\section{Protection of Human and Animal Subjects}

This was a quality improvement effort and as per our university guidelines exempt from review by our Institutional Review Board.

\section{Conflict of Interest}

None declared.

\section{References}

1 Evans RS. Electronic health records: Then, now, and in the future. Yearb Med Inform 2016(Suppl 1):S48-S61

2 Slight SP, Berner ES, Galanter W, et al. Meaningful use of electronic health records: experiences from the field and future opportunities. JMIR Med Inform 2015;3(03):e30

3 Overview of MACRA by Center for Medicare and Medicaid Services. Available at: https://qpp.cms.gov/. Accessed November 1, 2016

4 Bowens FM, Frye PA, Jones WA. Health information technology: integration of clinical workflow into meaningful use of electronic health records. Perspect Health Inf Manag 2010;7:1d

5 Pivovarov R, Elhadad N. Automated methods for the summarization of electronic health records. J Am Med Inform Assoc 2015;22 (05):938-947

6 Herasevich V, Ellsworth MA, Hebl JR, Brown MJ, Pickering BW. Information needs for the OR and PACU electronic medical record. Appl Clin Inform 2014;5(03):630-641

7 Vist A. Available at: https://www.va.gov/VA_Monograph_2019. pdf. Accessed June 9, 2017

8 Vist A. Imaging. Available at: https://www.va.gov/health/IMAGING/docs/VistA_Imaging_Dicom_Conformance_Statement.pdf. Accessed June 7, 2018

9 Cohen GR, Adler-Milstein J. Meaningful use care coordination criteria: perceived barriers and benefits among primary care providers. J Am Med Inform Assoc 2016;23(e1):e146-e151

10 Moon TS, Gonzales MX, Woods AP, Fox PE. Improving the quality of the operating room to intensive care unit handover at an urban teaching hospital through a bundled intervention. J Clin Anesth 2016;31:5-12

11 Sittig DF, Singh H. Electronic health records and national patientsafety goals. N Engl J Med 2012;367(19):1854-1860

12 Schonberger RB, Barash PG, Lagasse RS. The surgical care improvement project antibiotic guidelines: should we expect more than good intentions? Anesth Analg 2015;121(02):397-403

13 Wang J, Wongvibulsin S, Henry K, Fujita S. Quantifying and visualizing medication adherence in patients following acute myocardial infarction. AMIA Annu Symp Proc 2018;2017:2299-2303 